\title{
Universal amplitude ratios in finite-size scaling: three-dimensional Ising model
}

\author{
Martin Weigel and Wolfhard Janke \\ ${ }^{a}$ Institut für Theoretische Physik, Universität Leipzig, Augustusplatz 10/11, 04109 Leipzig, Germany
}

Motivated by the results of two-dimensional conformal field theory (CFT) we investigate the finite-size scaling of the mass spectrum of an Ising model on three-dimensional lattices with a spherical cross section. Using a cluster-update Monte Carlo technique we find a linear relation between the masses and the corresponding scaling dimensions, in complete analogy to the situation in two dimensions. Amplitude ratios as well as the amplitudes themselves appear to be universal in this case.

\section{INTRODUCTION}

Divergent correlation lengths of a statistical system approaching a critical point establish the symmetry of scale invariance on which finite-size scaling theory is based. In a continuum critical theory, however, there are additional symmetries present, namely translational, rotational and inversion invariance. The implications of the group of these symmetries, as explored in conformal field theory [1] [2], go far beyond simple scaling theory, especially for two-dimensional systems, where the whole content of scaling operators as well as for example higher correlation functions can be generically extracted from this symmetry.

As for a prominent result, conformal invariance suffices to determine the critical two-point function in the plane. Using a conformal (logarithmic) transformation that wraps the plane around an infinite-length cylinder one thus arrives at an expression for the correlation function of a primary (conformally covariant) operator on the cylinder [3], a geometry one generically considers in transfer matrix calculations. The fact that this transformation does only affect the radial but not the angular part of the coordinates led Cardy 㧮 to a generalization for the higher-dimensional geometries $S^{d-1} \times \mathbb{R}$. Thus, exactly as in two dimensions, the large distance exponential decay of correlations is determined by a correlation length that is given by the inverse of the scaling dimen- sion $x$ of the operator under consideration,

$\xi=\frac{R}{x}$

where $R$ is the radius of $S^{d-1}$. It has to be noted, however, that the meaning of a primary operator is a priori not well defined in three dimensions that this result has to be considered a conjecture and a numerical analysis is more than an exercise in this case.

A first attempt to establish such evidence using the Hamiltonian formulation of the Ising model and Platonic solids as discretization of the sphere $S^{2}$ was inconclusive due to the restricted number and size of regular polyhedra [6]. Using $a p$ proximate quadrangulations we are able to check Cardy's conjecture for systems sufficiently large to carry out a proper finite-size scaling (FSS) analysis.

\section{LATTICE DISCRETIZATION}

There are several straightforward choices for model lattices with spherical topology 7 ; the more natural are variants of a rectangular mesh on a cube. Different refinements can be applied to reduce the concentration of the curvature around the corners of the cube such as filling in triangles instead of the cube corners or projecting the cube on the sphere by varying the link lengths, i.e., applying an appropriate site-dependent weight func-

${ }^{1}$ For an ansatz for its definition see 5 and references therein. 
tion to the action. For those cube-like lattices, however, differences in the scaling behavior of bulk quantities are found to be quite small [7]. As for our observables, there is some evidence to believe that ratios of correlation lengths of primary operators are universal [8 10], so that we can expect good agreement regardless of the lattice used if Cardy's conjecture holds. For the amplitudes themselves one might find effects of the non-uniformity of the lattice, but would not expect them to become visible until a very high level of precision is reached. We thus here use the simple cubic approximation to the sphere by six $L \times L$ square lattices with suitably chosen boundary conditions; effects of the discretization will be considered elsewhere [11].

Having chosen a lattice approximation to the sphere one has to fix a definition of the radius $R$ of the sphere the cube with edge length $L$ should correspond to. As is easily checked the three possibilities of assigning a unit area to each lattice site, each pair of bonds, or each lattice square, respectively, lead to total lattice areas of

$A= \begin{cases}6 L(L-2)+8 & \text { "sites", } \\ 6 L(L-2)+6 & \text { "bonds", "squares", }\end{cases}$

and generate via the relation $R=\sqrt{A / 4 \pi}$ two different sorts of pseudo radii, which only differ by a constant shift, thus leading to slightly different approaches to the leading FSS amplitude.

\section{SIMULATIONAL DETAILS}

We consider a classical, ferromagnetic, nearestneighbor Ising model with Hamiltonian

$\mathcal{H}=-J \sum_{\langle i, j\rangle} s_{i} s_{j}, \quad s_{i}= \pm 1$

on lattices compound of the above described cubic discretizations of the sphere times a linear direction of length $L_{z}$, modelling the $\mathbb{R}$-direction. In order to minimize the effect of a finite $L_{z}$ it was (self-consistently) chosen so that $L_{z} / \xi \approx 15$ and periodic boundary conditions in $z$-direction were applied. Simulations were done using the Wolff single-cluster update algorithm at an inverse temperature $\beta=0.2216544$ (3) [12], checking for the influence of a temperature shift off the critical point by reweighting. To enable a proper FSS analysis system sizes ranging from $L=4$ to $L=12$ were used, corresponding to radii from about 2 to 8 and overall lattice volumes of 6000 to 300000 spins.

Since the densities of energy and magnetization are (the only) primary operators in the twodimensional Ising model and the lowest lying states of the two sectors, we here consider the amplitudes of the corresponding (exponential) correlation lengths $A_{\sigma / \epsilon}=\xi_{\sigma / \epsilon} / R$ and their ratio. Simulational measurements were done for the (connected) correlation functions $G^{c}(z)$ of these operators using a zero mode projection. Estimators for the correlation lengths that eliminate additive and multiplicative constants in the exponential decay are then given by

$\xi(z)=\Delta\left[\ln \frac{G^{c}(z)-G^{c}(z-\Delta)}{G^{c}(z+\Delta)-G^{c}(z)}\right]^{-1}$,

with $\Delta=1,2,3, \ldots$, from which overall estimates $\xi(R)$ are formed by averaging. A jackknife technique was used to reduce estimator bias and for the error analysis; for details see [10,13].

\section{NUMERICAL RESULTS}

Using the above described procedures one ends up with scaling plots for the correlation lengths as shown in Fig. 11(a) for the magnetization density that show an almost perfect linear behavior and invisible differences between the different radii definitions according to Eq. (2). Plotting the amplitudes $\xi / R$ (Fig. 1 (b)), however, reveals the presence of corrections to scaling that enforce the use of nonlinear fits of the form

$\xi(R)=A R+B R^{\alpha}$,

with a free parameter $\alpha$ that introduces a systematic error to the final amplitudes $A_{\sigma / \epsilon}$ due to higher order corrections. A combination of the fits for the two sorts of radii gives the amplitudes

$$
\begin{aligned}
A_{\sigma} & =1.996(20), \\
A_{\epsilon} & =0.710(38),
\end{aligned}
$$

which agree well with the conjectured amplitudes

$$
\begin{aligned}
& A_{\sigma}^{\text {conj }}=1 / x_{\sigma}=1.9324(19), \\
& A_{\epsilon}^{\text {conj }}=1 / x_{\epsilon}=0.70711(35),
\end{aligned}
$$



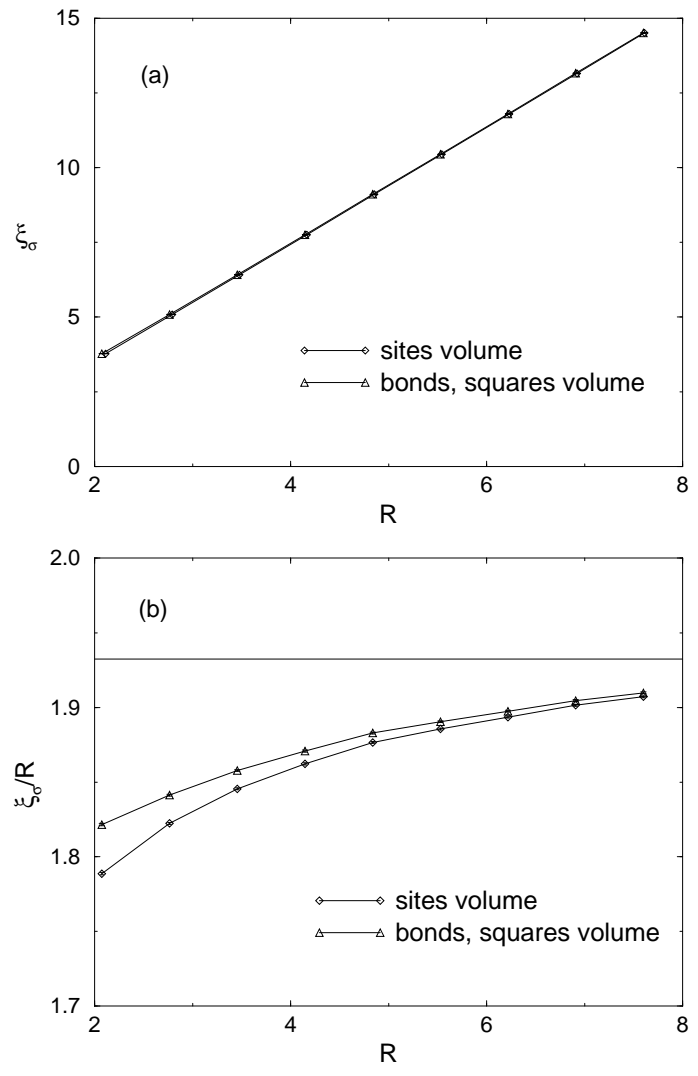

Figure 1. (a) FSS plot for the spin correlation length $\xi_{\sigma}(R)$. (b) Scaling of the amplitudes $\xi_{\sigma} / R$. The horizontal line indicates the conjectured amplitude according to Eq. (7).

taking into account the systematic error due to the different radii definitions that is of the same order of magnitude as the statistical error. The amplitude ratios compare as:

$$
\begin{aligned}
& A_{\sigma} / A_{\epsilon}=2.81(15), \\
& A_{\sigma}^{\text {conj }} / A_{\epsilon}^{\text {conj }}=x_{\epsilon} / x_{\sigma}=2.7326(16) \text {. }
\end{aligned}
$$

\section{CONCLUSIONS}

The presumably universal ratio of the amplitudes of the spin and energy correlation lengths of the Ising model on a cubic model of $S^{2} \times \mathbb{R}$ agrees with the inverse ratio of the scaling dimensions as conjectured by Cardy for the continuum case. Moreover, corrections to scaling due to the non- uniform distribution of curvature over the lattice that might influence the amplitudes themselves seem not to be very important at the given level of accuracy; only the slightly shifted result for the spin amplitude $A_{\sigma}$ might indicate the onset of such effects. In connection with the results for systems with toroidal cross section [10,13 this seems to indicate a deeper analogy between the $2 \mathrm{D}$ and $3 \mathrm{D}$ situations. In this latter case, however, the system is not conformally related to a flat space which renders impossible an analytical treatment of Cardy's kind. In the spherical case, on the other hand, conformal flatness is fulfilled, but the algebraic meaning of the primarity of an operator remains unclear. Thus our result shows that spin and energy densities of the 3D Ising model are primary operators, taking Eq. (1) as a definition of primarity in three dimensions.

MW thanks the Deutsche Forschungsgemeinschaft for support through the Graduiertenkolleg "Quantum Field Theory".

\section{REFERENCES}

1. J. L. Cardy, in Phase Transitions and Critical Phenomena, eds. C. Domb and J. L. Lebowitz, vol. 11 (Academic Press, 1987), p. 55 .

2. M. Henkel, Conformal Invariance and Critical Phenomena (Springer, 1999).

3. J. L. Cardy, J. Phys. A17 (1984) L385.

4. J. L. Cardy, J. Phys. A18 (1985) L757.

5. K. Lang and W. Rühl, Nucl. Phys. B402 (1993) 573.

6. F. C. Alcaraz and H. J. Herrmann, J. Phys. A20 (1987) 5735.

7. C. Hoelbling and C. B. Lang, Phys. Rev. B54 (1996) 3434.

8. M. Henkel, J. Phys. A20 (1987) L769.

9. M. A. Yurishchev, Phys. Rev. E55 (1997) 3915.

10. M. Weigel and W. Janke, Phys. Rev. Lett. 82 (1999) 2318.

11. M. Weigel and W. Janke, to be published.

12. A. L. Talapov and H. W. J. Blöte, J. Phys. A29 (1996) 5727.

13. M. Weigel and W. Janke, Ann. Phys. (Leipzig) 7 (1998) 575. 